\title{
Expresión tisular de proteínas reparadoras e infiltración linfocítica tumoral: significado pronóstico en el carcinoma colorrectal resecado
}

\author{
Tissular expression of mismatch repair proteins and tumour \\ lymphocytic infiltration: prognostic significance in resected \\ colorectal carcinoma
}

\author{
F. Borda ${ }^{1}$, J.M. Martínez-Peñuela ${ }^{2}$, A. Borda ${ }^{1}$, J. Urman ${ }^{1}$, J. Jiménez ${ }^{1}$, J.M. Zozaya ${ }^{1}$
}

\section{RESUMEN}

Fundamento. En el cáncer colorrectal se discute la posible relación entre la expresión patológica de proteínas reparadoras (EPPR) y la infiltración linfocítica tumoral (ILT), así como el posible efecto pronóstico de ambos factores.

Material y métodos. Se han revisado 243 cánceres colorrectales, resecados consecutivamente. Estudiamos inmunohistoquímicamente la EPPR de MLH1, MSH2 y MSH6. La ITL se valoró mediante la tinción de CD3 en el epitelio tumoral. Comparamos la mortalidad y progresión tumoral post-operatoria entre los casos con y sin EPPR y con y sin ITL. Adicionalmente estudiamos la mortalidad y progresión tumoral entre los casos EPPR (+), según presentaran o no ITL.

Resultados. El 13,6\% tumores expresaron EPPR (+) y el $25,5 \%$ ITL $(+)$. El seguimiento fue: $73,8 \pm 34,6$ meses. La frecuencia de ITL $(+)$ resultó similar entre tumores EPPR $(+)$ : $27,3 \%$ y EPPR (-): 25,2\% (p = 0,80). Los casos EPPR (+) mostraron menor mortalidad: $12,1 \%$ versus $23,3 \%(\mathrm{p}=0,15)$ y menor progresión tumoral: $21,2 \%$ versus $29 \%(\mathrm{p}=0,35)$. Las neoplasias ITL $(+)$ tuvieron menor mortalidad: $9,7 \%$ versus $26 \%[\mathrm{p}=0,007 ; \mathrm{OR}=3,27(1,25-9,05)]$ y progresión tumoral: $12,9 \%$ versus $33,1 \%$ [p $=0,002 ; \mathrm{OR}=3,35(1,42-8,15)]$. Los 9 tumores EPPR (+) e ILT (+) no presentaron mortalidad ni progresión tumoral, frente a una mortalidad: $16,7 \%$ y progresión: $29,2 \%$ de los 24 casos EPPR (+) e ITL (-) p = 0,19 y p $=0,07$ respectivamente.

Conclusiones. No se ha encontrado relación entre EPPR e ITL, con tasas muy similares de ILT $(+)$ entre casos con y sin EPPR. La ILT $(+)$ mostró un efecto pronóstico favorable superior a la EPPR $(+)$. La combinación de ILT $(+)$ e EPPR $(+)$ parece tener un efecto protector acumulativo, aunque su escasa frecuencia resta significación al hallazgo.

Palabras clave. Neoplasias colorrectales. Histopatología. Inmunohistoquímica. Pronóstico.Microsatélites.

\begin{abstract}
Background. In colorectal cancer there is discussion about the possible relation between the mismatch repair protein expression (MMRPE) and tumour lymphocytic infiltration (TLI), as well as the possible prognostic effect of both factors.

Methods. A review was made of 243 colorectal cancers, consecutively resected. We made an immunohystochemical study of the MMRPE of MLH1, MSH2 and MSH6. The TLI was evaluated through CD3 staining in the tumoural epithelium. We compared mortality and post-operative tumoural progression amongst the cases with and without MMRPE and with and without TLI. Additionally, we studied mortality and tumoural progression amongst MMRPE (+) cases, according to whether or not they presented TLI.

Results. Thirteen point six percent of the tumours expressed MMRPE (+) and 25.5\% TLI (+). The follow-up was: $73.8 \pm 34.6$ months. The frequency of TLI (+) turned out to be similar between MMRPE (+) tumours: $27.3 \%$ and MMRPE (-): $25.2 \%$ $(\mathrm{p}=0.80)$. The MMRPE $(+)$ cases showed less mortality: $12.1 \%$ versus $23.3 \%(p=0.15)$ and less tumoural progression: $21.2 \%$ versus $29 \%(\mathrm{p}=0.35)$. The ITL neoplasias $(+)$ had a lower mortality: $9.7 \%$ versus $26 \%$ [ $\mathrm{p}=0.007$; $\mathrm{OR}=3.27(1.25-9.05)$ ] and tumoural progression: $12.9 \%$ versus $33.1 \%[\mathrm{p}=0.002$; $\mathrm{OR}=3.35$ (1.42-8.15)]. The 9 MMRPE (+) and ILT (+) tumours did not present mortality or tumoural progression, against a mortality: $16.7 \%$ and progression: $29.2 \%$ of the 24 MMRPE (+) and TLI (-) cases $\mathrm{p}=0.19$ and $\mathrm{p}=0.07$ respectively.

Conclusions. No relation was found between MMRPE and TLI, with very similar rates of TLI (+) between cases with and without MMRPE. The TLI (+) showed a favourable prognostic effect higher than that of the MMRPE $(+)$. The combination of TLI (+) and MMRPE (+) seems to have an accumulative protective effect, although its limited frequency reduces the significance of the finding.
\end{abstract}

Key words. Colorectal neoplasias. Hystopathology. Immunohystochemistry. Prognosis. Microsatellites.
1. Servicio de Digestivo. Complejo Hospitalario de Navarra. Pamplona

2. Servicio de Anatomía Patológica. Complejo Hospitalario de Navarra. Pamplona

Recepción: 30 de marzo de 2012

Aceptación provisional: 10 de mayo de 2012

Aceptación definitiva: 19 de junio de 2012

\author{
Correspondencia: \\ Fernando Borda \\ C/ Olite, $37,7^{\circ}$ Izda \\ 31004 Pamplona \\ E-mail: bordafernando@yahoo.es
}




\section{INTRODUCCIÓN}

El cáncer colorrectal se origina, en un $85 \%$ de los casos, por la llamada vía supresora o de inestabilidad cromosómica, con activación de oncogenes como el K-ras y la inhibición de genes supresores (APC, DCC, TP53 $)^{1,2}$. Otra forma mucho menos frecuente de cancerogénesis se produce por la alteración del sistema de reparación de errores producidos durante la replicación del ADN, que está controlada por los genes MMR (mismatch repair), fundamentalmente los genes MLH1, MSH2, MSH6 y PSM2 ${ }^{3,4}$. Estas lesiones se denominan "tumores por inestabilidad de microsatélites" y suponen el 15\% de los cánceres colorrectales esporádicos y el 95\% de los cánceres colorrectales hereditarios no polipoideos o síndrome de $\mathrm{Lynch}^{3,4}$. Las alteraciones del mecanismo de reparación que caracteriza a los cánceres con inestabilidad de microsatélites pueden diagnosticarse mediante el estudio genético de las mutaciones germinales ${ }^{5,6}$, por análisis de microsatélites ${ }^{7}$ o por técnicas inmunohistoquímicas de expresión de las proteínas reparadoras ${ }^{8}$.

En su conjunto, el cáncer colorrectal sigue presentando unas importantes tasas de mortalidad, que en nuestra comunidad, suponen el 10,5\% del número total de fallecidos por cáncer en varones y el $14,7 \%$ en las mujeres ${ }^{9}$. Diversos estudios sugieren que las neoplasias por inestabilidad de microsatélites pueden presentar un pronóstico más favorable que el resto, sin que se conozcan exactamente los motivos ${ }^{10-12}$. Por otra parte, se asume que el cáncer colorrectal puede inducir una respuesta celular anti-tumoral, mediada por linfocitos $\mathrm{T}^{13}$. Esta respuesta inmune local del huésped frente al tumor se manifiesta en forma de una infiltración linfocítica tumoral (ILT) que, también según publicaciones recientes, se podría acompañar de un mejor pronóstico ${ }^{14,15}$.

La posible relación entre inestabilidad de microsatélites y la ILT está actualmente en discusión, con resultados discordantes en la bibliografía ${ }^{13,16-18}$. Por ello, hemos planteado el presente estudio, con el objetivo de determinar en una serie de cánceres colorrectales resecados la relación entre la frecuencia de expresión patológica de proteínas reparadoras (EPPR) y la ILT, valorada mediante estudio inmunohistoquímico del CD3 como pan-marcador de linfocitos $\mathrm{T}^{13}$. Así mismo, pretendemos analizar el posible valor pronóstico sobre la mortalidad y la progresión tumoral post-operatoria de la EPPR y la ILT, tanto de modo aislado como en el subgrupo de cánceres que presentasen expresión de ambos procesos.

\section{MATERIAL Y MÉTODOS}

Analizamos retrospectivamente una serie de 243 casos de cáncer colorrectal, operados consecutivamente. Tras la intervención, los pacientes fueron seguidos clínicamente y contestaron a una encuesta que incluía su autorización para analizar sus datos. La revisión histológica e inmunohistoquímica de las piezas de resección tumoral fue realizada por un único patólogo, con el fin de evitar variaciones inter-observador.

La EPPR y la ILT se estudiaron de modo cualitativo, con técnicas inmunohistoquímicas. Determinamos la expresión tisular patológica de MLH-1, MSH-2 y MSH-6 mediante la incubación de los cortes histológicos con sus anticuerpos primarios monoclonales, obtenidos de ratón, frente a la proteína humana (Biocare Medical, USA: clones G16815 , FE11 y BC/44, respectivamente), incluyendo un control positivo. Cualquier tinción nuclear fue clasificada como positiva. Consideramos como casos con expresión patológica de las proteínas reparadoras aquellos en los que al menos uno de los 3 marcadores mostró una tinción negativa.

La ILT se valoró mediante el estudio inmunohistoquímico de los linfocitos $\mathrm{T}$ intraepiteliales, expresados por el marcador CD3, empleando el anticuerpo monoclonal de ratón clon 2gv6 (Ventana Medical System, USA), incluyendo un control positivo. La tinción se valoró de modo cualitativo, mediante el análisis de 5 campos del epitelio tumoral y otros 5 de los bordes de la pieza de resección, libres de tumor. Se procedíó al contaje de los linfocitos CD3, obteniéndose las medias correspondientes. Se consideraron como positivos los casos en los que la tinción media de linfocitos T en el epitelio del tumor fue 3 veces superior a la del epitelio no tumoral de los bordes de la 
pieza quirúrgica, por campo de 20 aumentos, siempre que la cifra media de linfocitos CD3 en el epitelio del tumor fuese igual o superior a 4 .

Comparamos la frecuencia de EPPR y de ILT entre los diferentes estadios tumorales, según la clasificación pTNM.

Estudiamos la posible relación entre EPPR e ILT, comparando la frecuencia de ILT $(+)$ entre los casos con y sin EPPR.

Analizamos posibles diferencias en cuanto a mortalidad debida al cáncer, así como la progresión tumoral tras la resección quirúrgica, entre los casos EPPR $(+)$ y los EPPR (-). Igualmente, comparamos las tasas de exitus y de progresión tumoral entre los tumores con y sin ITL.

De modo adicional, estudiamos la mortalidad y progresión post-operatoria del tumor entre los casos $\operatorname{EPPR}(+)$, según presentasen o no ILT.

Para el estudio estadístico empleamos los tests de Fisher y Chi cuadrado, determi- nando la odds ratio (OR), con su índice de confianza al 95\% (IC). Consideramos significativos los valores de $\mathrm{p}<0,05$.

\section{RESULTADOS}

La serie estudiada estuvo compuesta por 167 hombres $(68,7 \%)$ y 76 mujeres (31,3\%). La edad media fue de 62,8 \pm 12 años. La clasificación pTNM de los cánceres fue la siguiente: estadio I = 26,7\%; II = 39,1\%; III $=25,1 \%$ y IV $=9,1 \%$.

Los tumores presentaron EPPR (+) en $33 / 243$ casos (13,6\%). La figura 1 representa la expresión normal y patológica (ausencia de tinción) de MLH-1. La ILT fue (+) en $62 / 243$ (25,5\%) (Fig. 2), presentando homogeneidad en cuanto a su distribución en el epitelio. No se registraron diferencias significativas entre el estadio tumoral pTNM y la frecuencia de EPPR ( $p=0,21)$, ni entre el pTNM y la presencia de ILT $(p=0,20)$.
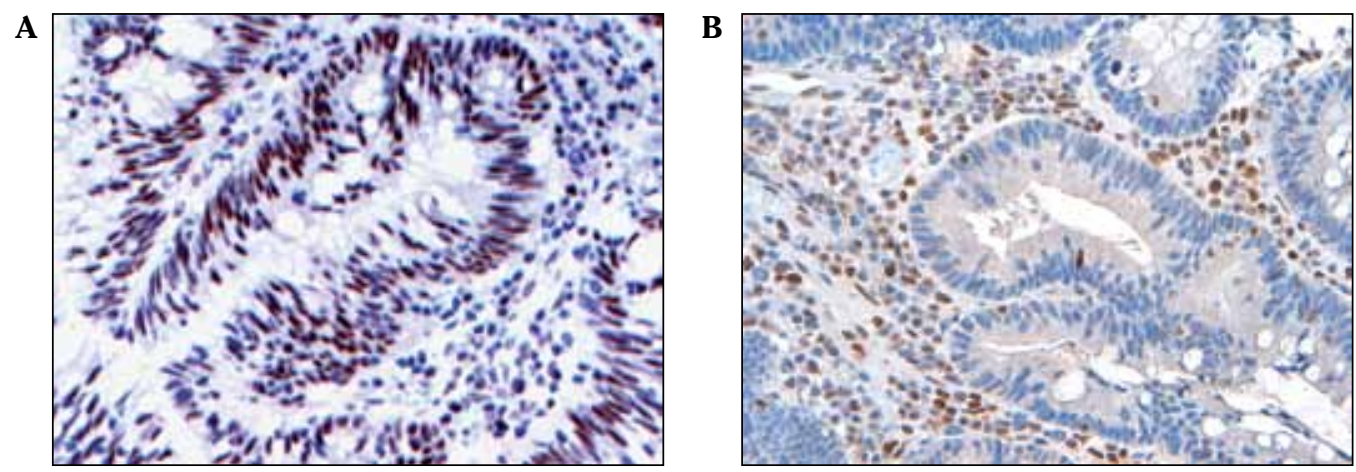

Figura 1. A: Expresión normal de la proteína reparadora MLH-1; B: expresión patológica (ausencia de tinción) de MLH-1.

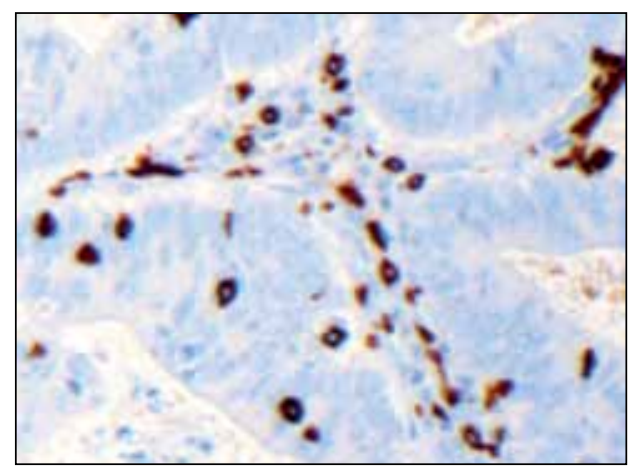

Figura 2. Expresión tisular positiva de linfocitos CD3 en el epitelio tumoral. 
No hemos objetivado relación entre expresión patológica de proteínas reparadoras e infiltración linfocítica tumoral, registrando una frecuencia muy similar de casos ITL(+) entre los tumores EPPR (+): 27,3\% y los EPPR (-): 25,2\% (p = 0,80).

Tras un seguimiento medio de $73,8 \pm$ 34,6 meses, registramos una mortalidad global del $21,8 \%$, y una progresión tumoral post-operatoria del $28 \%$. No encontramos diferencias en cuanto a la duración del seguimiento clínico entre los casos EPPR (+): $76 \pm 37,9$ meses y EPPR (-): 73,5 $\pm 34,1(\mathrm{p}=$
$0,70)$, ni entre el seguimiento de los tumores ILT (+): 75,8 $\pm 34,1$ e ITL (-): 73,2 $\pm 34,9$ meses $(p=0,61)$.

Con respecto al posible significado pronóstico de la expresión de proteínas reparadoras, observamos cómo los tumores EPPR (+) presentaron menor mortalidad: $12,1 \%$ que los EPPR (-): 23,3\%, sin alcanzar significación estadística $(\mathrm{p}=0,15)$. La progresión tumoral post-operatoria también fue menor en las neoplasias EPPR (+): $21,2 \%$ con respecto a las EPPR (-): $29 \%$ (p =0,35) (Fig. 3).

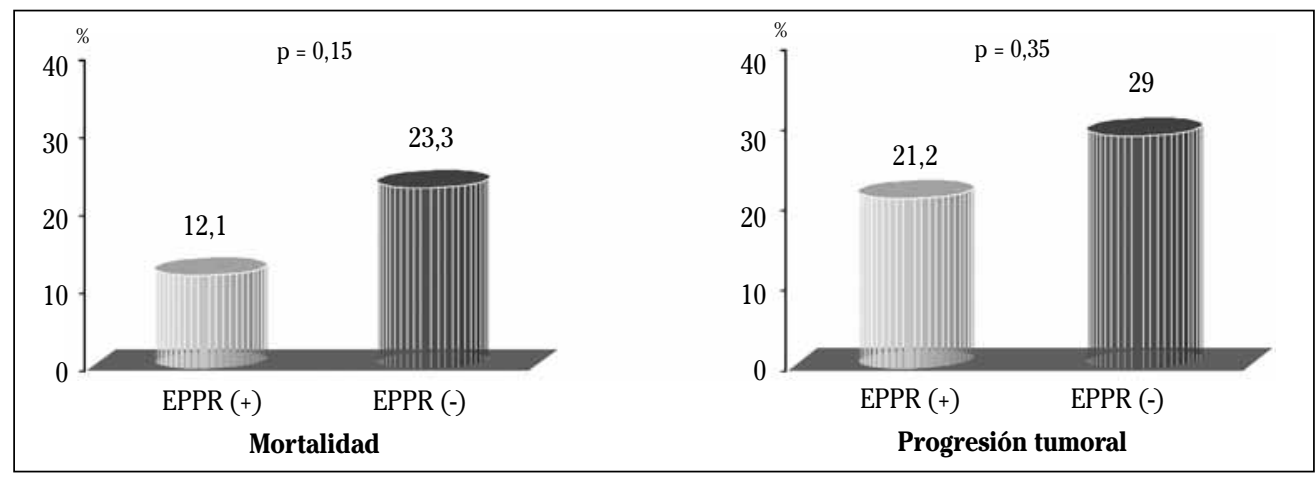

Figura 3. Mortalidad y progresión tumoral post-operatoria, según la expresión patológica de las proteínas reparadoras (EPPR).

La infiltración tumoral linfocítica se acompañó de una significativa menor mortalidad, ILT (+): 9,7\% versus ITL (-): $26 \%$ [p $=0,007 ; \mathrm{OR}=0,31(0,11-0,80)]$ con menor frecuencia de progresión tumoral ILT $(+)$ : $12,9 \%$ frente a ILT (-): $33,1 \%[\mathrm{p}=0,002 ;$ OR $=$ $0,30(0,12-0,70)]$ (Fig. 4).

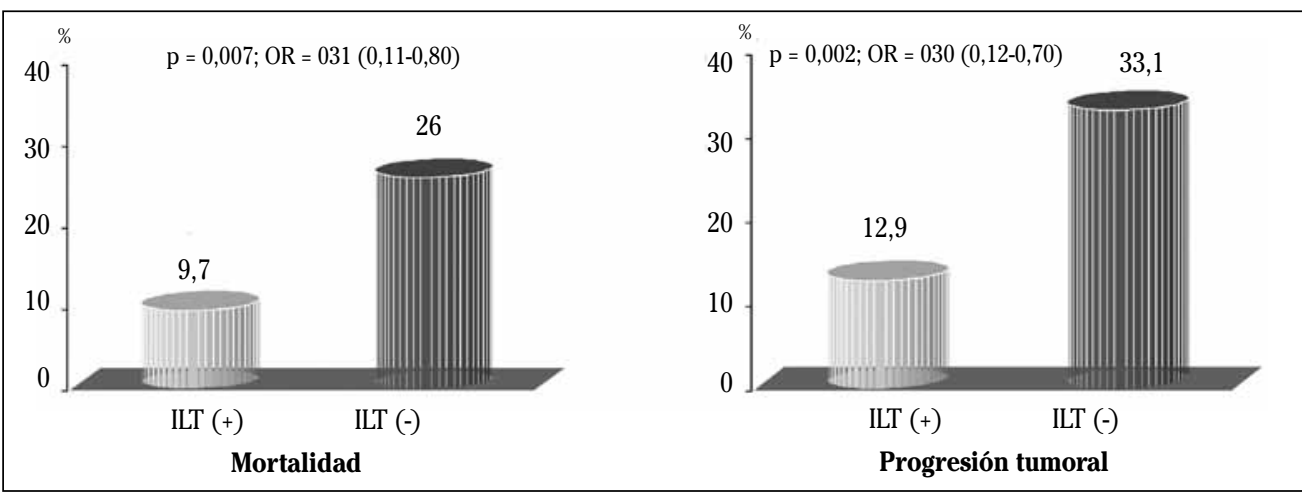

Figura 4. Mortalidad y progresión tumoral, en relación con la infiltración linfocítica tumoral (ILT). 
Finalmente, hemos estudiado el pronóstico del subgrupo de 33 pacientes con tumores EPPR (+), según presentasen o no infiltración linfocítica tumoral. Nueve de los 33 tumores EPPR (+) también fueron ILT (+). En estos 9 casos no registramos mortalidad ni progresión tumoral, frente a una mortalidad del $16,7 \%$ y una progresión tumoral del $29,2 \%$ de los 24 casos restantes ( $\mathrm{p}=0,19$ y $\mathrm{p}=0,07$ respectivamente).

\section{DISCUSIÓN}

Los cánceres colorrectales por inestabilidad de microsatélites están constituidos en una proporción muy minonitaria por el síndrome de Lynch o cánceres colorrectales hereditarios no polipoideos (2-3\% del total de los cánceres colorrectales) y fundamentalmente por el grupo de cánceres esporádicos con silenciamiento epigenético de la expresión de MLH1, por hipermetilación de su gen promotor ${ }^{19,20}$, alcanzando estos casos el 15\% del total de los carcinomas de colon y recto ${ }^{10-12}$.

El diagnóstico de los cánceres por inestabilidad de microsatélites se obtiene por el análisis genético de las mutaciones terminales ${ }^{5,6}$, o por el estudio de microsatélites, analizando las inserciones o delecciones del $\mathrm{ADN}$ tumoral ${ }^{21}$. Si bien estas técnicas son las más exactas, su mayor complejidad, menor disponibilidad y elevado coste, han propiciado el estudio inmunohistoquímico de la expresión tisular de las proteínas reparadoras: MLH1, MSH2, MSH6 y PMS2 como el método de aproximación para el estudio de los tumores por inestabilidad de microsatélites ${ }^{8,12,22,23}$. Esta técnica de tinción tisular, sencilla, económica y realizable en la gran mayoría de los Servicios de Anatomía Patológica está actualmente aceptada para el screening, habiéndose llegado a publicar unos valores de sensibilidad del $92-100 \%$, con una especificidad del $99-100 \%$ para los casos de cáncer colorrectal esporádico con inestabilidad de microsatélites ${ }^{21,23}$, aunque se acepta que la expresión conservada de las proteínas reparadoras puede omitir algún caso de mutación germinal en el síndrome de Lynch $^{23}$. Si bien otros trabajos valoran la inestabilidad de microsatélites determinando únicamente la expresión de las proteínas reparadoras MLH1 y MSH2 $2^{8,24}$, nosotros hemos incluido el análisis de MSH6. A pesar de ello, contamos con la limitación metodológica de no haber estudiado la proteína PMS2, aunque dicha limitación es más teórica que práctica, ya que la expresión patológica aislada de PMS2 es muy poco frecuente, alcanzando tan solo un $0,8 \%$ de los casos en una amplia serie multicéntrica nacional $^{21}$.

En la revisión bibliográfica efectuada, la mayoría de los trabajos registran un pronóstico más favorable en los casos con inestabilidad de microsatélites ${ }^{10-12}$, sin que todavía se conozcan suficientemente sus causas $^{12}$. La menor agresividad biológica de estos cánceres se ha atribuido a una menor prevalencia de mutaciones en el gen K-ras, o a la pérdida de heterocigosidad en los genes p53 o DCC, ya que todos estos factores implican un peor pronóstico ${ }^{12,25,26}$. Otra hipótesis en discusión es la que relaciona la inestabilidad de microsatélites con la ILT. En el cáncer colorrectal se producen péptidos inmunogénicos, que pueden ocasionar una respuesta anti-tumoral por parte del huésped ${ }^{27}$. Esta respuesta inmune focal está mediada por linfocitos T, dando lugar a una infiltración linfocítica tumoral, con destrucción de células neoplásicas y teórico mejor pronóstico para el paciente ${ }^{14,15}$. La posible relación entre inestabílidad de microsatélites e infiltración linfocitaria está en discusión, habiéndose publicado trabajos con resultados contradictorios. Así, unos autores refieren una mayor frecuencia de ILT en los tumores con inestabilidad de microsatélites ${ }^{16,17}$, mientras otros trabajos no confirman esta relación ${ }^{13,18}$.

Una posible explicación a estos resultados discordantes puede radicar en la diferente metodología empleada en los diversos estudios, tanto en las técnicas de diagnóstico de la inestabilidad de microsatélites como, especialmente, en el estudio de la ILT: métodos cuantitativos o cualitativos de contaje celular, subtipos de linfocitos $\mathrm{T}$ estudiados, localizacíón intraepitelial o estromal de los mismos, etc. ${ }^{12,13,14,16,18,28}$. En los estudios previos, la 
valoración de la infiltración linfocitaria se ha llevado a cabo por métodos muy diferentes. Estas técnicas varían desde el contaje de los linfocitos por autoanalizador de imagen que permiten obtener unos datos más reproducibles ${ }^{14,16}$, métodos de valoración semi-cuantitativa con elaboración de diferentes sistemas de puntuación ${ }^{13,18}$, hasta simples métodos cualitativos ${ }^{12,28}$.

En la literatura revisada no existe un acuerdo para precisar a partir de que punto se considera positiva la ILT. Los límites establecidos en los distintos estudios son muy variables: presencia de 5 linfocitos por campo de gran aumento en una tinción de hematoxilina-eosina ${ }^{12}$, más de 40 linfocitos CD3/1000 células epiteliales ${ }^{29}$, hasta la elaboración de "scores" tan imprecisos como grado 1: no linfocitos; grado 2: linfocitos esporádicos; grado 3: moderada presencia de linfocitos; grado 4: abundantes linfocitos ${ }^{18}$.

La valoración de la ILT por un método cualitativo es una técnica aceptada, que ha sido empleada por otros autores en publicaciones recientes ${ }^{12,28}$. En nuestro caso fue llevada a cabo por un único patólogo para evitar posibles diferencias inter-observador y empleando adicionalmente como control áreas no tumorales del mismo paciente, lo que aumenta la especificidad de la presencia de linfocitos en la neoplasia.

$\mathrm{Al}$ igual que otros trabajos, nosotros hemos analizado la expresión de CD3 como pan-marcador de la totalidad de los linfocitos $\mathrm{T}^{13}$. En los estudios publicados, la valoración de la infiltración linfocitaria por CD3 se ha efectuado a nivel del epitelio tumoral $^{13,16,22,28}$ y de diversas áreas del estroma $^{13,18,28}$. La frecuencia de linfocitos $\mathrm{T}$ es mayor en el estroma, lo que puede restar significación a su presencia ${ }^{28}$. Por este motivo y aunque los datos de la bibliografía son variables ${ }^{13,16,18,28}$, algunos autores aconsejan limitar su análisis al epitelio tumoral, dado que es esta localización la que muestra una mayor relación con el pronóstico del cáncer colorrectal ${ }^{22,28}$. En nuestro estudio hemos seguido esta indicación que, de modo añadido, reduce el trabajo para el patólogo.

En la serie analizada, la frecuencia de tumores con expresión patológica de proteínas reparadoras ha sido del 13,2\%, cifra similar a la publicada ${ }^{24}$. Igualmente, el porcentaje de casos con ILT (+) que hemos registrado: $25,5 \%$ concuerda con lo previamente descrito ${ }^{18}$. Estas frecuencias de EPPR y de ILT (+) no han mostrado diferencias significativas entre los distintos estadios tumorales que componen nuestra casuística.

Como ya hemos mencionado, se ha postulado una mayor proporción de casos con ILT entre los tumores con inestabilidad de microsatélites frente a los tumores estables $^{12,21}$. En nuestro estudio, y al igual que en otras publicaciones ${ }^{13,18}$, no hemos encontrado ninguna relación entre EPPR e ILT, habiendo registrado una frecuencia de casos con infiltración linfocitaria muy similar entre los tumores con alteración de las proteínas reparadoras: $27,3 \%$ y los que no presentaban dicha alteración: $25,2 \%$.

Tras la resección quirúrgica de la neoplasia en los tumores con alteración de las proteínas reparadoras se registra un mejor pronóstico, alcanzando a los seis años de la intervención unas cifras de mortalidad claramente inferiores: $12,1 \%$, con respecto a los casos sin expresión patológica de las proteínas reparadoras: $23,3 \%$. Igualmente el porcentaje de casos con progresión tumoral post-operatoria fue menor en las neoplasias con EPPR: 21,2\% versus 29\%. Aunque estos datos concuerdan con el pronóstico más favorable descrito en la literatura $^{10-12}$, el reducido número de casos de nuestra casuística que expresaron alteración de las proteínas reparadoras (33/243) ha podido influir en que nuestros resultados no alcancen significación estadística.

Por lo que respecta al valor pronóstico de la reacción inmune del huésped, expresado a través del infiltrado linfocitario tumoral, nuestros datos apoyan la hipótesis de una evolución más favorable en los tumores ILT (+), con una tasas de mortalidad y de progresión significativamente inferiores con respecto a los ILT (-), presentando unos riesgos tres veces inferiores.

Los resultados que hemos obtenido indican, por una parte, que en el cáncer colorrectal resecado la infiltración linfocitaria constituye un factor pronóstico favorable, 
con un efecto más importante sobre la mortalidad y la progresión tumoral que el de la alteración de las proteínas reparadoras. En segundo término, la falta de relación observada entre la EPPR y la ILT no apoya la teoría de que la inestabilidad de microsatélites induzca una mayor respuesta inmune anti-tumoral y sugiere que el mecanismo de protección observado en ambos procesos pudiera producirse por mecanismos diferentes, como ya se había sido sugerido en otros trabajos ${ }^{12,18}$.

De modo adicional, hemos querido estudiar el posible efecto protector añadido de la expresión simultánea de EPPR e ILT $(+)$, recientemente propuesto ${ }^{29,30}$. A diferencia de las neoplasias con EPPR pero sin infiltración linfocitaria, en el subgrupo de 9 pacientes con EPPR e ILT (+) no registramos ningún caso de fallecimiento ni de progresión tumoral post-operatoria. No obstante, el escaso número de cánceres que cumplen ambas condiciones resta significación a este hallazgo, que sugiere un posible efecto beneficioso acumulativo $\mathrm{y}$ que debiera ser confirmado por otros estudios con una casuística más amplia.

\section{BIBLIOGRAFÍA}

1. Fearon ER, Volgestein B. A genetic model for colorectal tumorigenesis. Cell 1990; 61: 759767.

2. Smith G, Carey FA, Beattie J, Sales M, Kernohan NM, SмIтн G et al. Mutations in APC, Kirstenras, and p53-alternative genetic pathways to colorectal cancer. Z Gastroenterol 2003; 42: 201-203.

3. SorëIDe K, Janssen EAM, SÖIland H, KöRner H, BAAK JPA. Microsatellite instability in colorectal cancer. Br J Surg 2006; 93: 395-406.

4. Lanza G, Gafì R, Santini A, Maestri I, Guerzoni L, CAVAZZINI L. Immunohistochemical test for MLH1 and MSH2 in stage II and III colorectal cancer patients. J Clin Oncol 2006; 24: 23592367.

5. Ponz de Leon M, Benatti P, Di Gregorio C, PedroNI M, Losi L, GenuaRdi M et al. Genetic testing among high-risk individuals in families with hereditary nonpoyposis colorectal cancer. Br J Cancer 2004; 90: 882-887.

6. Papp J, Kovacs ME, Olah E. Germline MLH1 and $\mathrm{MSH} 2$ mutational spectrum including frequent large genomic aberrations in Hungarian hereditary non-polyposis colorectal cancer families: implications for genetic testing. World Gastroenterol 2007; 13: 2727 2732.

7. Piñol V, Castells A, Andreu M, Castellví-Bel S, Alenda C, Llor X et al. Accuracy of revised Bethesda guidelines, microsatellite instability, and immunochemistry for the identification of patients with hereditary nonpolyposis colorectal cancer. JAMA 2005; 293: 1986-1994.

8. Warrier SK, Trainer AH, Lynch AC, Mitchell C, HISCOCK R, SAWYER S et al. Preoperative diagnosis of Lynch syndrome with DNA mismatch repair immunohistochemistry on a diagnostic biopsy. Dis Colon Rectum 2011; 54: 1480-1487.

9. Ardanaz E, Moreno-Iribas C, Pérez de Rada ME, EzPonda C, Floristán Y, NaVAridas N et al. Incidencia y mortalidad por cáncer en Navarra. Evolución en los últimos 30 años. An Sist Sanit Navar 2007; 30: 245-270.

10. Popat S, Hubner R, Houlston RS. Systematic review of microsatellite inestability and colorectal cancer prognosis. J Clin Oncol 2005; 23: 609-618.

11. Guastadisegni C, Colafranceschi M, Ottini L, DoGLIOTTI E. Microsatellite instability as a marker of prognosis and response to therapy: a meta-analysis of colorectal cancer survival data. Eur J Cancer 2010; 46: 2788-2798.

12. Chang EY, Dorsey PB, Frankhouse J, LeE RG, Walts D, Johnson W et al. Combination of microsatellite instability and lymphocytic infiltrate as a prognostic indicator in colon cancer. Arch Surg 2009; 144: 511-515.

13. Deschoolmeester V, Bayy M, Van Marck E, Weyler J, Vermeulen P, LaRdon F et al. Tumor infiltrating lymphocytes: an intriguing player in the survival of colorectal cancer patients. BMC. Immunol 2010; 11: 19-30.

14. Halama N, Zoernig I, Spille A, Michel S, Kloor M, Grauling-Halama S et al. Quantification of prognostic immune cell markers in colorrectal cancer using whole slide imaging tumor maps. Anal Quant Cytol Histol 2010; 32: 333340 .

15. Galon J, Costes A, Sanchez-Cabo F, Kirilovsky A, Miecnik B, Lagorce-Pagès C et al. Type, density, and location of immune cells within human colorectal tumors predict clinical outcome. Science 2006; 31: 1960-1964.

16. Nosho K, Baba Y, Thaka N, Shima K, Hayashi M, MEYERHARDT JA et al. Tumor-infiltrating T-cells subsets, molecular changes in colorectal cancer, and prognosis: cohort study and literature review. J Pathol 2010; 222: 350-366. 
17. Baker K, Zlobec I, Tornillo L, Terracciano L, JASS JR, LuGLI A. Differential significance of tumour infiltrating lymphocytes in sporadic mismatch repair deficient versus proficient colorectal cancers: a potential role for dysregulation of the transforming growth factorbeta pathway. Eur J Cancer 2007; 43: 624-631.

18. Dahlin AM, Henriksson ML, Van Guelpen B, StenLing R, Oberg A, Rutegard J et al. Colorectal cancer prognosis depends on T-cell infiltration and molecular characteristics of the tumor. Mod Pathol 2011; 24: 671-682.

19. Cunningham JM, Christensen ER, Tester DJ, Kim CY, Roche PC, Burgart LJ et al. Hypermethylation of the hMLH1 promoter in colon cancer with microsatellite instability. Cancer Res 1998; 58: 3455-3460.

20. Kuismanen SA, Holmberg MT, Salovaara R, DE la Chapelle A, Peltomäki P. Genetic and epigenetic modification of MLH1 accounts for a major share of microsatellite-unstable colorectal cancers. Am J Pathol 2000; 15: 1773-1779.

21. Payá A, Alenda C, Jover R, Aranda FI. Cáncer colorrectal con alteración de la vía reparadora. Claves para su identificación y relevancia clínica. Rev Esp Patol 2006; 39: 201-208.

22. Naito Y, Saito K, Shiba K, Ohuchi A, Saigenji K, NAGURA $\mathrm{H}$ et al. CD8+ T cells infiltrated within cancer cell nests as a prognostic factor in human colorectal cancer. Cancer Res 1998; 58: 3491-3494.

23. Ruszkiewicz A, Bennett G, Moore J, Manavis J, Rudzki B, Shen L et al. Correlation of mismatch repair genes immunohistochemistry and microsatellite instability status in HNPCC-associated tumours. Pathology 2001; 34: 541-547.
24. Ohrling K, Edler D, Hallström M, Ragnhammar $P$. Mismatch repair protein expression in an independent prognostic factor in sporadic colorectal cancer. Acta Oncol 2010; 49: 797804.

25. Forster S, Sattler HP, Hack M, Romanakis K, RoHDE V, SEITz G et al. Microsatellite instability in sporadic carcinomas of the proximal colon: association with diploid DNA content, negative protein expression of p53, and distinct histomorphologic features. Surgery 1998; 123: 13-18.

26. JASS JR, Biden KG, Cummings MC, Simms LA, WaLSH M, Schoch E et al. Characterisation of a subtype of colorectal cancer combining features of the suppressor and mild mutator pathways. J Clin Pathol 1999; 52: 455-460.

27. Linnebacher M, Gebert J, Rudy W, Woerner S, YuAN YP, Bork P et al. Frameshift peptidederived T-cell epitopes: a source of novel tumor-specific antigens. Int J Cancer 2001; 93: 6-11.

28. Sinicrope FA, Rego RL, Ansell SM, Knutson KL, FosTER NR, SARGENT DJ. Intraepithelial effector (CD3+)/regulatory (FoxP3+) T-cell ratio predicts a clinical outcome of human colon carcinoma. Gastroenterology 2009; 137: 12701279 .

29. JASS JR. Pathology of hereditary non polyposis colorectal cancer. Ann N Y Acad Sci 2000; 910: 62-73.

30. Kumar S, Chang Ey, Frankhouse J, Dorsey PB, LeE RG, Johnson N. Combination of microsatellite instability and lymphocytic infiltrate as a prognostic indicator for adjuvant therapy in colon cancer. Arch Surg 2009; 144: 835-840. 